Ann. Biol. anim. Bioch. Biophys., I974, 14 (4-B), 855-859.

\title{
EFFETS TOXIQUES D'UNE INTUBATION UNIQUE DE DIFFÉRENTES FRACTIONS PRÉPARÉES A PARTIR D'HUILES CHAUFFÉES CHEZ LE JEUNE RAT
}

\author{
B. POTTEAU et A. GRANDGIRARD \\ avec la collaboration technique de Geneviève Compoint et Suzanne Gratron \\ Station de Recherches sur la Qualité des Aliments de l'Homme, I. N. R. A., \\ 21034 Dijon Cedex
}

\section{RÉSUMÉ}

Chez le jeune Rat à jeun, l'administration d'une dose unique ( $\mathrm{I} \mathrm{ml}$ pour Ioo g de poids corporel) d'une fraction contenant les composés non complexables à l'urée obtenus à partir d'huiles de lin et de colza chauffées à $275^{\circ} \mathrm{C}$ sous azote ou à $200^{\circ} \mathrm{C}$ à l'air, provoque des effets toxiques très nets : mortalité élevée ou morbidité évidente, stéatose hépatique chez la plupart des survixants

Chez le jeune Rat à jeun, SHue et al. (1968) ont montré que l'administration d'une dose unique de composés concentrés par fractionnement (acides non complexables à l'urée) à partir de corps gras dégradés par la chaleur et l'oxydation, provoque une stéatose hépatique. Ils ont également mis en évidence qu'une administration répétée de cette fraction provoque une mortalité importante dans un délai inférieur à 7 jours. Ce dernier effet a également été obtenu par Nolen et al. ( $\left.\mathrm{r}^{6} 67\right)$ avec des fractions provenant de corps gras ayant subi des opérations de friture. Dans un travail antérieur, (Porreau et Nouvelor, 1974) nous avons obtenu une stéatose hépatique marquée en administrant, par intubation stomacale, de l'huile de lin chauffée à $275^{\circ} \mathrm{C}$ pendant I 2 heures sous atmosphère inerte; l'accumulation de lipides au niveau du foie atteint un maximum chez le jeune Rat maintenu à jeun $\mathbf{r} 8$ heures après l'intubation. Nous avons cherché à compléter ce précédent travail, d'une part en essayant de préciser dans quelle fraction se trouvent les composés responsables de la stéatose hépatique et de la toxicité, d'autre part en le généralisant au cas de plusieurs huiles riches en acide linolénique soumises à différentes conditions de chauffage. 
A cet effet, nous avons fait chauffer de l'huile de lin, de l'huile de colza et de l'huile de canbra soit à $275^{\circ} \mathrm{C}$ sous atmosphère inerte (azote), soit à $200^{\circ} \mathrm{C}$ en présence d'air, comme il est précisé dans le tableau I. Ces diverses huiles ont été saponifiées, l'insaponifiable a été éliminé et les acides ont été transformés en esters méthyliques; ceux-ci ont été ensuite séparés par la méthode de complexation à l'urée en deux fractions :

$\mathrm{C}=$ Esters complexables à l'urée ;

$\mathrm{F}=$ Esters non complexables à l'urée (filtrat).

Des précisions sur le mode de fractionnement et sur les caractéristiques de ces fractions sont données par ailleurs (Potteau et Dubois, 1974).

Dans une première série d'essais, les fractions ont été administrées par intubation stomacale à des rats mâles Wistar à l'âge du sevrage, à raison de $\mathbf{I} \mathrm{ml}$ pour roo $\mathrm{g}$ de poids corporel. Les animaux avaient été au préalable mis à jeun pendant 2 heures, et ils sont restés sans nourriture pendant $\mathrm{I} 8$ heures après l'intubation. Ils ont été alors sacrifiés, le foie a été pesé et congelé jusqu'à l'analyse. L'extraction des lipides totaux du foie a été effectuée selon la technique de Folch et al. (I957). Les résultats sont reportés sur le tableau I.

On voit que les animaux recevant des esters méthyliques d'huile de lin fraîche (témoins) ou la fraction $\mathrm{C}$ des esters complexables à l'urée provenant soit de l'huile de lin fraîche, soit des huiles de lin chauffées, ont tous survécu 18 heures après l'intubation et présentaient une teneur en lipides hépatiques de l'ordre de 5 à $6 \mathrm{p}$. 100 .

Par contre, les animaux recevant les fractions $\mathrm{F}$ des esters non complexables à l'urée se distinguent des précédents, selon les deux critères envisagés :

- soit par suite d'une létalité ou d'une morbidité évidente I 8 heures après l'intubation. C'est le cas pour les rats ayant reçu les fractions $\mathrm{F}$ provenant des huiles de lin chauffées à $275^{\circ} \mathrm{C}$ ou à $200^{\circ} \mathrm{C}$ et des huiles de canbra chauffées à $200^{\circ} \mathrm{C}$;

- soit par suite d'une stéatose hépatique marquée (plus de 7 p. Ioo de lipides totaux) chez les animaux survivants recevant la fraction $F$ provenant des huiles de lin, de colza et de canbra chauffées à $275^{\circ} \mathrm{C}$ et les huiles de colza chauffées à $200^{\circ} \mathrm{C}$. Une stéatose hépatique est également évidente chez les animaux recevant les esters méthyliques non fractionnés des huiles de lin chauffées à $275^{\circ} \mathrm{C}$ et à $200^{\circ} \mathrm{C}$.

Dans une deuxième série d'essais portant sur un nombre réduit de fractions, mais sur un nombre plus élevé d'animaux par lot, les intubations sont effectuées comme il a été indiqué plus haut, mais les rats ne sont pas sacrifiés I 8 heures après l'intubation. Ils reçoivent une provende commerciale et restent en observation pendant $\mathbf{z} 2$ jours. Les résultats sont reportés sur le tableau 2. On voit qu'une partie des animaux recevant les fractions $F$ provenant des huiles de lin chauffées meurent au cours des 3 premiers jours qui suivent l'intubation. Aucun des animaux témoins, ayant reçu des esters méthyliques préparés à partir d'huile d'arachide non chauffée, n'est mort. Il est frappant de constater que le chauffage à $200^{\circ} \mathrm{C}$ à l'air (plus proche des conditions usuelles de friture) cause une mortalité nettement plus élevée que le chauffage réalisé à $275^{\circ} \mathrm{C}$ sous atmosphère inerte. Par ailleurs, chez les animaux survivants qui ont reçu les fractions $F$, le gain de poids corporel en $\mathbf{r} 2$ jours est plus faible, et le poids du foie au terme des essais est plus élevé que chez les témoins.

En conclusion, comme SHUE et al. (1968) et Nolen et al. (1967), nous avons mis en évidence le caractère toxique de certaines fractions d'acides gras concentrés par la méthode de complexation à l'urée Iorsqu'elles sont administrées par intubation gastrique au rat sevré. Il convient cependant de mettre l'accent sur quelques différences essentielles entre ces deux travaux antérieurs et le nôtre :

I. NOLEN et al. (1967) avaient éliminé par distillation l'ensemble des produits polymérisés qui n'ont pas été testés dans leurs essais physiologiques. Dans les essais de SHue et al. (1968) et les nôtres, les produits polymérisés n'ont pas été éliminés des fractions administrées aux animaux.

2. Dans ces deux travaux antérieurs, les fractions avaient été obtenues à partir de corps gras ne contenant pas, ou très peu, d'acide linolénique avant leur dégradation thermo-oxydative. 
TOXICITÉ DE FRACTIONS D'HUILES CHAUFFÉES

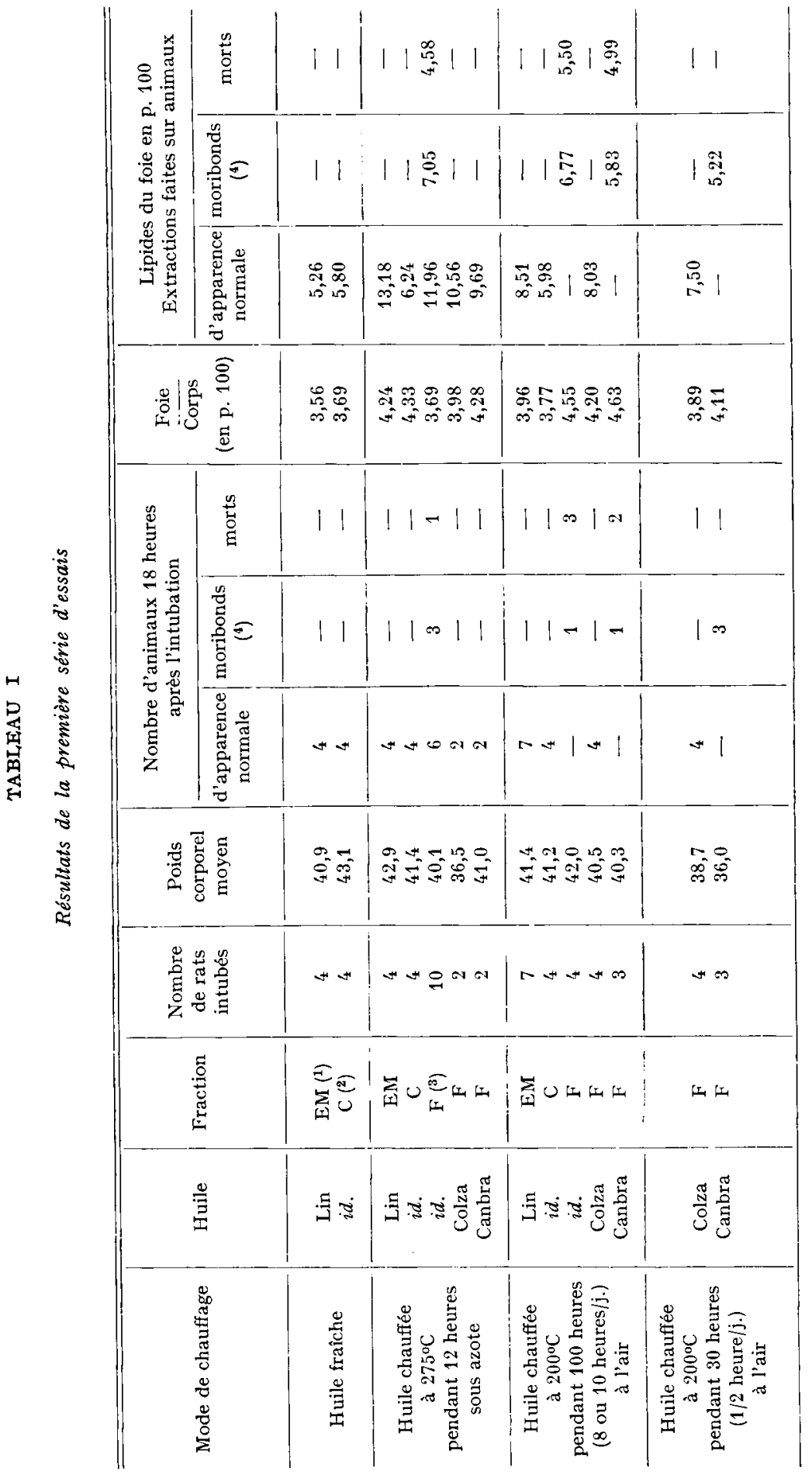

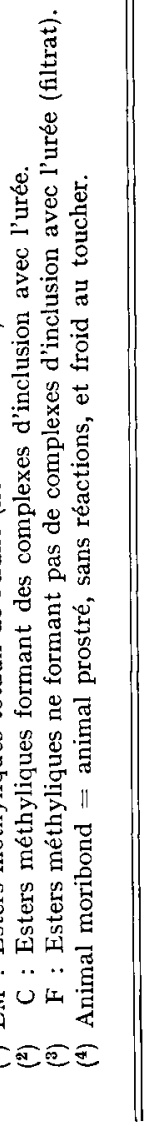


B. POTTEAU, A. GRANDGIRARD

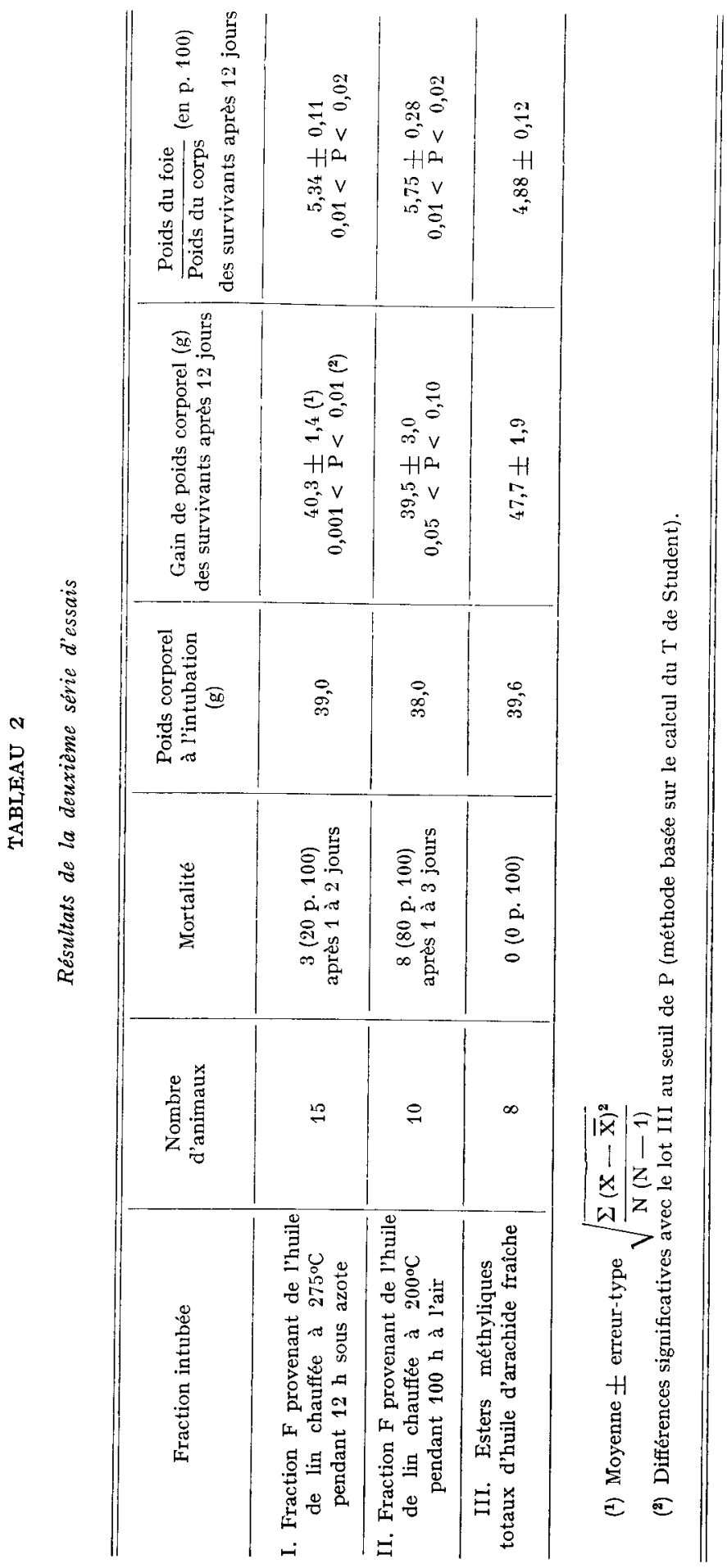


Les huiles utilisées dans le présent travail présentaient des teneurs en acide linolénique de l'ordre de 8 p. roo (colza, canbra) à 56 p. ıoo (lin).

3. Les signes les plus apparents de toxicité (létalité) étaient observés après une administration journalière répétée des fractions testées dans les essais de ShuE et al. (2 jours consécutifs) et de Nolen et al. ( 3 jours consécutifs). Dans la présente étude, une administration unique de certaines fractions nous a permis de mettre en évidence une létalité chez une partie importante des animaux, et d'autres signes de perturbations, en particulier au niveau du foie, chez les survivants.

Recu pour publication en juillet 1974.

\author{
SUMMARY \\ TOXIC EFFECTS ON THE YOUNG RAT OF A SINGIE INTUBATION \\ OF DIFFERENT FRACTIONS PREPARED FROM HEATED OILS
}

Young fasted rats are given a single dose ( $\mathrm{I} \mathrm{ml}$ for $\mathrm{roo} \mathrm{g}$ of body weight) of a fraction containing components which do not form complexes with urea. These components are obtained from linseed and rapeseed oils heated to $275^{\circ} \mathrm{C}$ under nitrogen or to $200^{\circ} \mathrm{C}$ in air. The single dose given has very clear toxic effects, such as high mortality or obvious morbidity and hepatic steatose, in most of the survivors.

\title{
RÉFÉRENCES BIBLIOGRAPHIQUES
}

Folch J., Lees M., Sloane-Stanley G. H., I957. A simple method for the isolation and purification of total lipids from animal tissues. J. Biol. Chem., 226, 497-509.

Nolen G. A., Alexander J.C., Artman N. R., I967. Long-term Rat feeding study with used frying fats. $J$. Nutr., 93, 337-348.

Potteau B. et Nouvelot A., 1974. (en préparation).

Potteau B. et Dubois P., I974. (en préparation).

Shue G. M., Douglass C. D., Firestone D., Friedman L., Friedman L., Sage J. S., I968. Acute physiological effects of feeding rats non-urea-adducting fatty acids (urea filtrate). $J$. Nutr., 94, I 7I-I77. 